\title{
Saddle Points Stability in the Replica Approach Off Equilibrium
}

\author{
M.E. Ferrero and M.A. Virasoro \\ May 4, 2018 \\ Dipartimento di Fisica, Università di Roma, La Sapienza, \\ I-00185 Roma, Italy \\ and \\ INFN Sezione di Roma I, Roma, Italy.
}

\begin{abstract}
We study the replica free energy surface for a spin glass model near the glassy temperature. In this model the simplicity of the equilibrium solution hides non trivial metastable saddle points. By means of the stability analysis performed for one and two real replicas constrained, an interpretation for some of them is achieved.
\end{abstract}




\section{Introduction}

In spin glass models, replica symmetry breaking implies the existence of many pure states of equilibrium. Their hierarchical organisation is described by the order parameter $Q_{a b}$ [1]. In the SK model $Q_{a b}$ has a continuum form and it is a marginally stable saddle point of the replica free energy surface in the limit $n \rightarrow 0$ [2]. In other models, like the p-spin spherical model, the stable solution is just one-step, yielding a simpler equilibrium state organisation [3].

In this paper we investigate this different feature from the stability point of view. We use the generalisation of the replica method recently developed to study non equilibrium states [4] [5]. The general idea is to consider $R$ identical copies of the model (real replicas) constrained to have specific mutual overlaps. Then, by forcing the overlaps out of equilibrium, one can probe the phase-space structure of the original model and obtain information on some of its non equilibrium states.

By performing the stability analysis in the replica approach off equilibrium, we uncover the physical meaning for some saddle points other than the one yielding the Gibbs-measure results.

\section{The unconstrained model and its saddle points}

\section{$2.1 \quad$ The model}

Let us consider the following truncated replica free energy density

$$
F(Q)=-\frac{1}{n}\left(t \operatorname{Tr} Q^{2}+\frac{1}{3} \operatorname{Tr} Q^{3}+\frac{1}{6} \sum_{a \neq b} q_{a b}^{3}+\frac{y}{12} \sum_{a \neq b} q_{a b}^{4}\right)
$$

where we consider $t \sim T_{c}-T$ to be small. The cubic term $\sum q_{a b}^{3}$, absent in the SK-model, gives a $y$ independent replica symmetry breaking. A term of this kind appears in the p-spin spherical model and in the Potts model [6]. The $y$ parameter will play the role of a control parameter. We will assume it to be small $(y<<t)$ but positive or negative (in the SK model it is positive).

In [6] it was shown that for $y<0$ the stable solution is simply a matrix with one replica symmetry breaking (1RSB). The probability distribution of the overlap between equilibrium configurations is then

$$
P_{e q}(q)=m_{1} \delta(q)+\left(1-m_{1}\right) \delta\left(q-q_{E A}\right)
$$

where $q_{E A}=2 t+\frac{10}{3} y t^{2}$ and $m_{1}=\frac{1}{2}+y t$ are obtained by stationary conditions. 
In the solution we will consistently truncate the $O\left(y^{2}\right)$ terms. As a consequence of the stationary conditions, the $O\left(y^{2}\right)$ terms in the solution give $O\left(y^{4}\right)$ contribution to free energy. In the following we will then consider free energy up to $O\left(y^{3}\right)$.

\subsection{Unstable saddle points}

For positive $y$ we know that a better approximation to the full solution is given by the 2RSB saddle point

$$
\begin{aligned}
q(x) & = \begin{cases}q_{0}=0 & 0<x<m_{1} \\
q_{1}=t+\frac{23}{12} y t^{2} & m_{1}<x<m_{2} \\
q_{2}=2 t+\frac{23}{6} y t^{2} & m_{2}<x<1\end{cases} \\
m_{1} & =\frac{1}{2}+\frac{1}{2} y t \\
m_{2} & =\frac{1}{2}+\frac{3}{2} y t
\end{aligned}
$$

and free energy $F_{2 R S B}>F_{1 R S B}\left(F_{2 R S B}-F_{1 R S B} \sim y^{2}\right)$.

To study fluctuations around this 2RSB S.P. we consider the eigenvalue equation

$$
\left(2 t+q_{a b}+y q_{a b}^{2}+\lambda\right) f_{a b}+\{Q, f\}_{a b}=0
$$

where $f=Q-Q_{S P}$. This equation, analysed in details in the context of the SK model [7], can be solved through the explicit construction of the eigenvectors exploiting the symmetry of the equation. In the notation of [7] the eigenvectors are classified as longitudinal $\left(f_{a b}\right)$, anomalous $\left(f_{a b}^{\mu}\right)$ and replicon $\left(f_{a b}^{\mu \nu}\right)$. For positive $y$ the minimum eigenvalue is $\lambda=-\frac{y t^{2}}{6}$ and it is well known that unstable directions belong also to the replicon family.

Let us discuss the analytic continuation to negative $y$ of this solution, that we expect to have new unstable directions. It leads to $m_{2}<m_{1}$, a result which does not allow the usual probabilistic interpretation.

We found the minimum eigenvalue to be negative $\left(\lambda=\frac{5 y t^{2}}{6}\right)$. The unstable eigenvectors belong to the longitudinal and anomalous families. They are

$$
\text { longitudinal } \rightarrow f_{a b}=\left\{\begin{array}{lll}
f_{0}(0)=0 & \text { if } & a \cap b=0 \\
f_{0}(1) \neq 0 & \text { if } & a \cap b=1 \\
f_{0}(2) \neq 0 & \text { if } & a \cap b=2
\end{array}\right.
$$




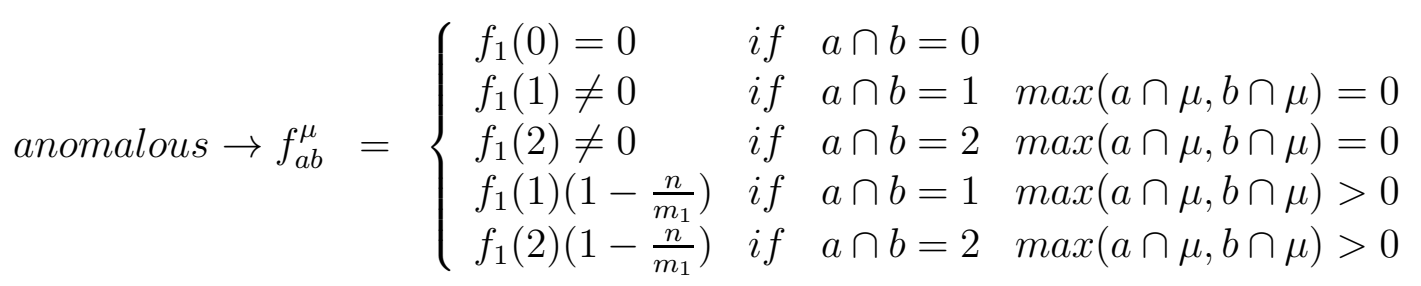

where, as in [7], $a \cap b=k$ if $q_{a b}=q_{k}$.

For future reference, we anticipate that by considering additional RSB the new unstable S.P. will have $m_{i+1}-m_{i} \sim y$. There is, of course, the analytic continuation (to negative $y$ ) of the continuum solution (slope $=\frac{1}{y}$ ), which is the only one marginally stable for positive $y$. It has obviously a free energy greater than the other solutions.

\section{The constrained model}

Let us consider $R$ Real Replicas and introduce in the partition function $\frac{R}{2}(R-1)$ complex Lagrangean multipliers $\left(\epsilon_{r s}\right)$ to keep the mutual overlaps constrained $\left(q_{c}^{r s}\right)$. The effective averaged free energy has two contributions [4]

$$
F_{R}\left(\mathbf{Q}, q_{c}^{r s}\right)=F(\mathbf{Q})+F_{\text {constr }}\left(\epsilon_{a}^{r s}, q_{c}^{r s}\right)
$$

The functional $F(\mathbf{Q})$ has the same form as for a single real replica with $\mathbf{Q}$ a $R n \times R n$ matrix

$$
\mathbf{Q}=\left(\begin{array}{cccc}
Q^{11} & P^{12} & \ldots & P^{1 R} \\
P^{12^{T}} & Q^{22} & \ldots & P^{2 R} \\
\ldots & \ldots & \ldots & \ldots \\
P^{1 R^{T}} & P^{2 R^{T}} & \ldots & Q^{R R}
\end{array}\right)
$$

where $P_{a a}^{r s}=\epsilon_{r s}^{a a}$. The "interaction" term $F_{\text {constr }}\left(\epsilon_{a}^{r s}, q_{c}^{r s}\right)$ reads

$$
F_{\text {constr }}\left(\epsilon_{a}^{r s}, q_{c}^{r s}\right)=-\frac{\beta^{2}}{2} \sum\left(q_{c}^{r s}-\epsilon_{a}^{r s}\right)^{2}
$$

The stationary conditions are then

$$
\frac{\partial F}{\partial q_{a b}^{r r}}=0 \quad \frac{\partial F}{\partial p_{a b}^{r s}}=0 \quad \frac{\partial F}{\partial p_{a a}^{r s}}=\beta^{2}\left(p_{a a}^{r s}-q_{c}^{r s}\right)
$$


In the following we consider the particular case $R=2$ and we make for $Q$ and $P$ a Parisi ansatz. Before analysing the solution, let us explain how we studied the stability.

\subsection{RR Stability}

To study the Hessian of $F_{2}$ we consider $F$ at fixed $p_{a a}=p_{d}$. From (9) we see that the saddle point for $p_{a a}$, which originally is integrated along the imaginary axis, lies then on the real axis. The integration path can be deformed to go through the saddle point but the integration path will be perpendicular to the real axis. This S.P. has to a maximum when compared to values along the integration path. Notice that this distinguish $p_{d}$ from all other matrix terms which are integrated along the real axis. The stability with respect to $p_{d}$ fluctuations around the saddle point can be then verified in (8) and, being not proportional to $t$, decouples.

The equation for $F_{2}$ is then

$$
\left(2 t+\mathbf{q}_{\alpha \beta}+y \mathbf{q}_{\alpha \beta}^{2}+\lambda\right) \mathbf{f}_{\alpha \beta}+\{\mathbf{Q}, \mathbf{f}\}_{\alpha \beta}=0
$$

where $\alpha \beta=1, \ldots, 2 n$ but $\alpha-\beta \neq n$. In order to reduce this problem to an ultrametric one we can observe that the symmetry group of the Hamiltonian without constraint is $S_{2 n}$ (all replicas are equivalent). After imposing the constraint it becomes $S_{n} \otimes$ $\left(S_{2}\right)^{\otimes n}$ (to permute simultaneasly replicas in both systems or to permute equivalent replicas between systems). Since all solutions found are symmetrical under the action of $S_{2}$ on all the replicas, we divide the eigenvectors in two superfamilies, the symmetrical and the antisymmetrical one. We then parametrise the fluctuation matrix $\mathbf{f}$ around the $\mathbf{Q}_{S . P \text {. }}$ in terms of

$$
\mathbf{f}=\left(\begin{array}{cc}
+f^{1} & +f^{x} \\
+f^{x} & +f^{1}
\end{array}\right)+\left(\begin{array}{cc}
+f^{z} & +f^{y} \\
-f^{y} & -f^{z}
\end{array}\right)
$$

where the $f^{1, x, z}$ are $n * n$ symmetrical matrices and $f^{y}$ antisymmetrical.

The eigenvalue equations are now decoupled and read, for $a \neq b$,

$$
\begin{aligned}
& \left(2 t+q_{a b}+y q_{a b}^{2}+\lambda\right) f_{a b}^{1}+\left\{Q, f^{1}\right\}_{a b}+\left\{f^{x}, P\right\}_{a b}=0 \\
& \left(2 t+p_{a b}+y p_{a b}^{2}+\lambda\right) f_{a b}^{x}+\left\{P, f^{1}\right\}_{a b}+\left\{f^{x}, Q\right\}_{a b}=0
\end{aligned}
$$

and 


$$
\begin{aligned}
& \left(2 t+q_{a b}+y q_{a b}^{2}+\lambda\right) f_{a b}^{z}+\left\{Q, f^{z}\right\}_{a b}+\left[f^{y}, P\right]_{a b}=0 \\
& \left(2 t+p_{a b}+y p_{a b}^{2}+\lambda\right) f_{a b}^{y}+\left\{Q, f^{y}\right\}_{a b}+\left[f^{z}, P\right]_{a b}=0
\end{aligned}
$$

The eigenvectors of (12) and (13) are then respectively of the form

$$
\begin{aligned}
& \mathbf{f}=\left(\begin{array}{cc}
+f^{1} & +f^{x} \\
+f^{x} & +f^{1}
\end{array}\right) \mathbf{f}_{\mu}=\left(\begin{array}{ll}
+f_{\mu}^{1} & +f_{\mu}^{x} \\
+f_{\mu}^{x} & +f_{\mu}^{1}
\end{array}\right) \quad \mathbf{f}_{\mu \nu}=\left(\begin{array}{cc}
+f_{\mu \nu}^{1} & +f_{\mu \nu}^{x} \\
+f_{\mu \nu}^{x} & +f_{\mu \nu}^{1}
\end{array}\right) \\
& \mathbf{f}=\left(\begin{array}{cc}
+f^{z} & 0 \\
0 & -f^{z}
\end{array}\right) \quad \mathbf{f}_{\mu}=\left(\begin{array}{cc}
+f_{\mu}^{z} & +f_{\mu}^{y} \\
-f_{\mu}^{y} & -f_{\mu}^{z}
\end{array}\right) \quad \mathbf{f}_{\mu \nu}=\left(\begin{array}{cc}
+f_{\mu \nu}^{z} & +f_{\mu \nu}^{y} \\
-f_{\mu \nu}^{y} & -f_{\mu \nu}^{z}
\end{array}\right)
\end{aligned}
$$

where $f_{\mu}^{y}$ and $f_{\nu \mu}^{y}$ are antisymmetrical matrices breaking the hierarchical subgroups of permutation group (as the 1RR ones).

\subsection{RR constrained saddle points}

We investigate the possible $2 \mathrm{RSB}$ solutions in the range $0<p_{d}<q_{E A} p_{d}=p_{a a}^{12}$. We try to follow the solution by continuity when $p_{d}$ varies. At different points in the interval a stable solution becomes unstable and we have to search for the new stable solution.

Let us consider $y<0$. We find four critical $p_{d}$ values $0<p^{A}<p^{B}<p^{C}<$ $p^{D}<q_{E A}$. At each critical $p_{d}$ value, two different solutions coincide and zero modes appear to modify their stability.

We start at $p_{d} \simeq 0$. The stable solution is

$$
\begin{aligned}
q(x) & = \begin{cases}0 & 0<x<m_{2} \\
q_{E A}=2 t+\frac{10}{3} y t^{2} & m_{2}<x<1\end{cases} \\
p(x) & = \begin{cases}0 & 0<x<m_{2} \\
p_{d} & m_{2}<x<1\end{cases} \\
m_{2} & =\frac{1}{2}+y t
\end{aligned}
$$

and the free energy density is 


$$
F^{0 A}-F_{1 R S B}^{u n c o n s t r}=\Delta F^{0 A}=\frac{1}{12}\left(-2 y t^{2} p_{d}^{2}-p_{d}^{3}\right)+O\left(y^{4}\right)
$$

If $p_{d}=0$ the free energy is the same as the equilibrium one and $\frac{\partial F^{0 A}}{\partial p_{d}}=\frac{d F^{0 A}}{d p_{d}}=0$. For small $p_{d}$ the free energy is an increasing function of $p_{d}$ (fig.1). The minimum eigenvalue is $\lambda=-\frac{2}{3} y t^{2}-p_{d}$. Thus when $p_{d}>p^{A}=-\frac{2}{3} y t^{2}$ this solution becomes unstable and the new stable solution is:

$$
\begin{aligned}
q(x) & = \begin{cases}q_{0}=0 & 0<x<m_{1} \\
q_{1}=p_{d}-\frac{2}{3} y t\left(p_{d}-t\right) & m_{1}<x<m_{2} \\
q_{2}=2 t+\frac{10}{3} y t^{2}-\frac{1}{6} y p_{d}\left(p_{d}-4 t\right) & m_{2}<x<1\end{cases} \\
p(x) & = \begin{cases}p_{0}=0 & 0<x<m_{1} \\
p_{1}=p_{d}-\frac{2}{3} y t\left(p_{d}-t\right) & m_{1}<x<m_{2} \\
p_{2}=p_{d} & m_{2}<x<1\end{cases} \\
m_{1} & =\frac{1}{4}+y \frac{p_{d}}{4} \\
m_{2} & =\frac{1}{2}+y\left(t+\frac{p_{d}}{2}\right)
\end{aligned}
$$

The free energy is

$$
\Delta F^{A B}=\frac{y^{2}}{18}\left(2 p_{d} t^{4}-2 p_{d}^{2} t^{3}+p_{d}^{3} t^{2}-\frac{1}{4} p_{d}^{4} t\right)+O\left(y^{3}\right)
$$

This solution crosses, at $p_{d}=p^{A}$, the previous one. For $p_{d} \sim-y$ the minimum eigenvalue is $\lambda=\frac{p_{d}}{2}+\frac{y t^{2}}{3}$. As can be seen in (fig.11) and (fig.2), $\Delta F^{A B}$ is an increasing function until $p_{d}=p_{\max }=t+\frac{23}{12} y t^{2}$, where $\frac{d F^{B}}{d p_{d}}=\frac{\partial F^{A B}}{\partial p_{d}}=0$. For future reference we rewrite (18) on this specific value

$$
\begin{aligned}
q(x) & = \begin{cases}q_{0}=0 & 0<x<m_{1} \\
q_{1}=t+\frac{23}{12} y t^{2} & m_{1}<x<m_{2} \\
q_{2}=2 t+\frac{23}{6} y t^{2} & m_{2}<x<1\end{cases} \\
p(x) & = \begin{cases}p_{0}=0 & 0<x<m_{1} \\
p_{1}=t+\frac{23}{12} y t^{2} & m_{1}<x<1\end{cases} \\
m_{1} & =\frac{1}{4}+y \frac{t}{4} \\
m_{2} & =\frac{1}{2}+y \frac{3 t}{2}
\end{aligned}
$$


The minimum eigenvalue is $\lambda=-\frac{y t^{2}}{6}$. As $p_{d}$ increases further, $\Delta F^{A B}$ begins to decrease while all eigenvalues read positive until the second critical value $p^{B}$. The minimum eigenvalue for $p_{d} \simeq p^{B}$ is $\lambda=-y\left(p_{d}^{2}-8 p_{d} t+8 t^{2}\right) / 6$ and it corresponds to fluctuations of $q_{1}$ and $p_{1} \cdot p^{B}$ is defined by $\left.\lambda\right|_{p^{B}}=0$.

For $p_{d}$ greater than $p^{B}$ the stable solution becomes

$$
\begin{aligned}
q(x) & = \begin{cases}q_{0}=0 & 0<x<m_{1} \\
q_{1}=p_{d}-\frac{1}{9} y\left(-p_{d}^{2}+14 p_{d} t-14 t^{2}\right) & m_{1}<x<m_{2} \\
q_{2}=2 t+y \frac{10}{3} t^{2}-\frac{p_{d} y}{6}\left(p_{d}-4 t\right) & m_{2}<x<1\end{cases} \\
p(x) & = \begin{cases}p_{0}=0 & 0<x<m_{1} \\
p_{1}=p_{d}-\frac{1}{9} y\left(p_{d}^{2}-2 p_{d} t+2 t^{2}\right) & m_{1}<x<m_{2} \\
p_{2}=p_{d} & m_{2}<x<1\end{cases} \\
m_{1} & =\frac{1}{4}+y \frac{p_{d}}{4} \\
m_{2} & =\frac{1}{2}+y\left(t+\frac{p_{d}}{2}\right)
\end{aligned}
$$

The free energy of this solution $\left(\Delta F^{B C}\right)$, which differs from (18) by terms of $O\left(y^{3}\right)$, is decrasing in $p_{d}$ (fig2). Again at $p_{d}=p^{B}$ the two solutions crosses each other. The minimum eigenvalue is $\lambda=y\left(p_{d}^{2}-8 p_{d} t+8 t^{2}\right) / 6$. We have $p_{1}-q_{1}=$ $-2 y\left(p_{d}^{2}-8 p d t+8 t^{2}\right) / 9 \sim \lambda$ and the free energy difference between (18) and (21), expressed in terms of (21), reads $\sim(p 1-q 1)^{3} \sim-y^{3}$.

We found (21) to be stable for $p^{B}<p_{d}<p^{C} \simeq 3 / 2 t$. In the range $p^{C}<p_{d}<$ $p^{D}<q_{E A}$ we did'nt find any 2RSB stable solution. In particular (21) is unstable with respect to replicon fluctuations. We expect that additional RSB lead to the stable solution.

Thus, let us consider $p_{d}>p^{D}=2 t+\frac{23 y t^{2}}{6}$. The stable solution is

$$
\begin{aligned}
q(x) & = \begin{cases}0 & 0<x<m_{1} \\
\frac{2}{3}\left(p_{d}+t\right)+y \frac{10}{81}\left(p_{d}+t\right)^{2} & m_{1}<x<1\end{cases} \\
p(x) & =q(x) \\
m_{1} & =\frac{1}{4}+y \frac{1}{6}\left(p_{d}+t\right)
\end{aligned}
$$

The free energy, expressed in term of $\delta=p_{d}-q_{E A} \sim y$, reads (fig.3)

$$
\Delta F^{D q_{E A}}=\frac{1}{18}\left(-2 \delta^{2} t^{2} y-\delta^{3}\right)+O\left(y^{4}\right)
$$

and the minimum eigenvalue is $\lambda=\frac{4 \delta-2 y t^{2}}{3}$. 
Clearly for positive $y$ these solutions are just approximations to the full solution. In particular we found (20) to have the same instability than the 2RSB free solution $\left(\lambda=-\frac{y t^{2}}{6}\right)$.

\section{Discussions}

Let us analyse the previous results. For $p_{d}=0$ the two real replicas lie on configurations belonging to different equilibrium states. As the multiplicity of pure states give no contributions to the entropy, the free energy per replica is equal to that of the unconstrained system and $q(x)=q(x)^{\text {unconstr }}$ and $p(x)=0$. IF $p_{d}$ increases we force the overlap between couple of replicas to be out of equilibrium. The free energy increases until the maximum is reached at $p^{\max }$ while if $p_{d}>p^{\max }$ the free energy falls until $p_{d}$ of order of $q_{E A}$, where we are choosing configurations inside the same state. Since the overwhelming majority of pairs of configurations inside a state are at distance $q_{E A}$ from each other, if $p_{d}=q_{E A}$ we have twice the energy and twice the intra-state entropy.

At $p_{d}=p^{\max }$ we have $\frac{\partial F^{A B}}{\partial p_{d}}=\frac{d F^{A B}}{d p_{d}}=0$. The constraining force is then zero (on the S.P.) and the 2 real replicas are unconstrained. In fact the solution (20) is related through a replica permutation to the solution (3), because $m_{1}^{2 R R}=m_{1}^{1 R R} / 2$. Then $F_{\max }^{2 R R}=F_{2 R S B}^{1 R R}$. However the constraint still acts on the fluctuations and prevents the 2 replicas from sliding along the unstable direction.

It is remarkable that in this way we have been able to interpret an unstable 2RSB. We conjecture that with more constraints one will be able to stabilise solutions with more levels of breaking. Therefore the linear solution, which is the limit for an infinite number of breakings, will reappear.

The Parisi parameter $q(x)$ plays two different roles in the SK model. On the one hand it appears in the Gibbs measure and determines the $P_{e q}(q)$. In this case $q(x)$ must be necessarily monotonous. On the other hand $x(q)$ also appears in the Cavity Method where monotonicity does not seem to be required. It remains as an intriguing open question whether in systems as the present a non monotonous $x(q)$ could be relevant. Perhaps solving the $\mathrm{R}$ real replicas by a generalisation of the Cavity Method could elucidate this issue.

\section{Acknowledgements}

We are grateful to S. Franz, I. Kondor, J. Kurchan and T. Temesvari for useful discussions. 


\section{References}

[1] For a recent exposition on mean field theory of Spin Glasses see for example: M. Mézard, G. Parisi and M.A. Virasoro, "Spin Glass Theory and Beyond" (1987) (Singapore:World Scientific).

[2] C. de Dominicis and I. Kondor, Eigenvalues of the stability matrix for Parisi solution of the long-range spin-glass, Physical Review, 27(1):606-608, 1982.

[3] A. Crisanti, H. Horner and H.H. Sommers, The spherical p-spin interaction spin glass model: the statics, Z. fur Phys., B 87:341, 1992.

[4] S. Franz, G. Parisi, and M.A. Virasoro, The replica method on and off equilibrium, Journal de Physique (France), 2:1869, 1993.

[5] J. Kurchan, G. Parisi, and M.A. Virasoro, Barriers and Metastable States as Saddle Points in the Replica Approach, Journal de Physique, 3:1819, 1993.

[6] D.J. Gross, I.Kanter, and H.Sompolinsky, Mean-field theory of the potts glass, Physical Review, 55(3):304-307, 1985.

[7] C. de Dominicis and I. Kondor, Ultrametricity and zero modes in the shortrange Ising spin glass, Europhysics Letters, 2(8):617-624, 1986. 
Figure 1: $\Delta F$ as a function of $p_{d}$ plotted for $0<p_{d}<2 p^{A}, t=0.05$ and $y=-0.01$.

Figure 2: $\Delta F$ as a function of $p_{d}$ plotted for $0<p_{d}<q_{E A}, t=0.05$ and $y=-0.01$.

Figure 3: $\Delta F$ as a function of $p_{d}$ plotted for $p^{D}$ near $q_{E A}, t=0.05$ and $y=-0.01$. 